\title{
Multidetector Computed Tomography Evaluation of Mediastinal Lesions with Histopathological Diagnosis
}

\author{
Venkateshwaran Arumugam1, Hajimohammed Nazir², Konduru Varadarajulu Rajasekar1 \\ ${ }^{1}$ Department of Radiodiagnosis, Meenkashi Medical College and Research Institute, Kancheepuram, India \\ ${ }^{2}$ Department of Radiodiagnois, Sri Muthukumaram Medical College and Research Institute, Mangadu, India \\ Email: Venkateshwaran81@gmail.com, Hajimohammed867@yahoo.co.in, roshoraju@gmail.com
}

Received 8 May 2015; accepted 22 June 2015; published 25 June 2015

Copyright (C) 2015 by authors and Scientific Research Publishing Inc.

This work is licensed under the Creative Commons Attribution International License (CC BY). http://creativecommons.org/licenses/by/4.0/

(c) (i) Open Access

\begin{abstract}
The evaluation of mediastinal abnormalities is a challenging radiographical problem. Crosssectional imaging of the mediastinum by computed tomography now demonstrates precise anatomic details and is the imaging modality of choice for most mediastinal lesions. The following study was undertaken with the objectives of determining the mediastinal lesions affecting the mediastinum and to correlate the computed tomography findings with the histopathology reports. In this study, 50 patients clinically suspected with mediastinal mass lesions or who had chest radiograph with a suspicious mediastinal lesion were evaluated from October 2013 to September 2014. In our study, we used varying computed tomography features of each mediastinal pathology types to predict a histological diagnosis of mediastinal lesions. Most of the lesions could be predicted with good sensitivity, specificity and diagnostic accuracy.
\end{abstract}

\section{Keywords}

Mediastinal, MDCT, Enchancement, Mass

\section{Introduction}

The evaluation of mediastinal abnormalities is a challenging radio graphical problem. Cross-sectional imaging of the mediastinum by computed tomography [CT] now demonstrates precise anatomic details and is the imaging modality of choice for most mediastinal lesions. With the ability of high resolution MDCT scans to precisely localize lesions and biopsy needles, along with the delineation of adjacent structures, diagnostic fine-needle aspiration or core biopsy for both benign and malignant disease processes has become a quite safe and highly ac- 
curate procedure. The present study is to evaluate the mediastinal mass lesions on MDCT, differentiate based on their location and to correlate the MDCT findings with histopathology.

\section{Material \& Methods}

Study was done in Department of Radiodiagnosis, meenakshi medical college, kancheepuram, tamilnadu. The Radiology department is equipped with 1.5 Tesla MRI machine, 6 slice CT Scan, advanced USG machines, IITV, CR Mammography and digital radiography 0.50 patients clinically suspected with mediastinal mass lesions or who had chest radiograph with a suspicious mediastinal abnormality and referred to the Department of Radiodiagnosis, are taken up for the study. The study includes patients referred from various departments, both inpatient and outpatients. There is no limitation of age. The inclusion criteria were clinically and radiographically suspected cases of mediastinal mass lesions and Patients in whom chest radiograph showed incidental findings of mediastinal mass lesions.The Exclusion criteria were Traumatic causes, Cardiac causes and Cases in which, a tissue sampling could not be done.

\section{Discussion and Results}

The study was undertaken with the objectives of determining the mediastinal lesions affecting the mediastinum and to correlate the CT findings with the histopathology reports. On CT, mediastinal lesions are initially classified based on their location (i.e. anterior/middle/posterior). Based on the CT findings like nature of lesion, origin, calcification and post contrast enhancement, differential diagnosis is narrowed. Associated findings like mass effect, mediastinal invasion, pleural involvement, chest wall involvement and spine involvement are also taken into consideration. Finally, taking into account the age, clinical symptoms and CT findings, a CT diagnosis is finalized, which is later, correlated with histopathology diagnosis. Among the 50 cases, 26 cases (52\%) underwent CT guided biopsy (Figure 1) of the lesion. CT diagnosis of oesophageal carcinoma lesions underwent endoscopic biopsy. One case of oesophageal leiomyoma underwent endoscopic ultrasound guided biopsy. Middle mediastinal lymph node masses in paratracheal and subcarinal location underwent transbronchial needle aspiration. In remaining cases, histopathology was obtained from surgical resection specimen.

In our study of 50 cases of mediastinal lesions, $62 \%$ were malignant and $38 \%$ were benign. Malignant lesions were majority in our study. Benjamin et al. examined 215 patients with mediastinal masses over a 20-year period, among which $60 \%$ were benign and $40 \%$ were malignant [1]. In a study by Murray et al. on 132 patients, $85 \%$ were malignant and $15 \%$ were benign mediastinal masses [2].

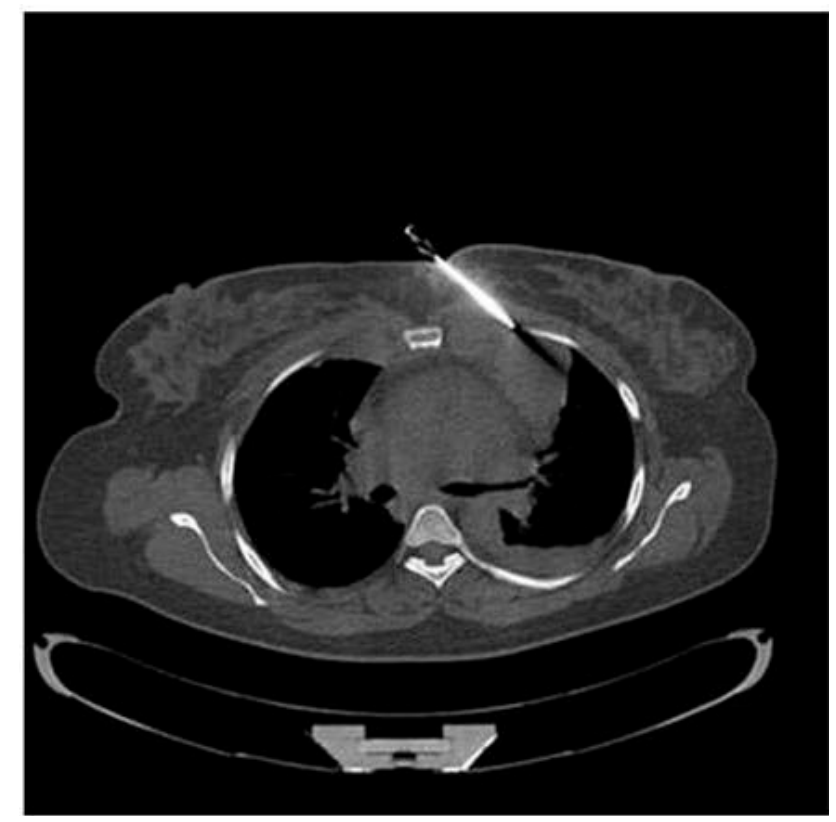

Figure 1. CT guided bispsy. 
In our study of 50 cases, the majority of the mediastinal masses were in the anterior mediastinum (Table 1) constituting $62 \%$ followed by middle (Table 2) and posterior mediastinum (Table 3), which were $44 \%$ and $18 \%$ respectively. It is similar to the study conducted by Strollo et al. in 1997 wherein anterior mediastinum constituted 50\% of the masses [3]. In Japan, Takeda et al. retrospectively reviewed 806 patients with mediastinal mass in a single institute between 1951 and 2000, in which anterior compartment was common location in adults and posterior mediastinum in children [4]. In their study, thymomas (36\%), germ cell tumors (16\%), congenital cysts (14\%), lymphomas (12\%), neurogenic tumors (11\%), and thyroid tumors (4\%) were seen in adult patients. Neurogenic tumors (46\%), germ cell tumor (19\%), lymphoma (13\%), congenital cysts (8\%), and thymomas (4\%) were seen in the pediatric patients. In our study the number of pediatric cases were very less. We had only 5 cases belonging to pediatric age group (i.e. up to 18 years of age) and which included 3 cases of lymphoma $(60 \%)$, one case of hemorrhagic thymic cyst (20\%) and one case of metastases (20\%). Majority of our cases belong to age group above 60 years which constituted $34 \%$.

Table 1. Anterior mediastinal lesion distribution.

\begin{tabular}{ccc}
\hline Type of lesion & Count & Percentage \\
\hline Thymoma & 5 & 16.1 \\
Invasive thymoma & 1 & 3.2 \\
Thymic carcinoma & 1 & 3.2 \\
Hemorrhagic thymic cyst & 1 & 3.2 \\
Multinodular goiter & 3 & 9.7 \\
Thyroid lymphoma & 1 & 3.2 \\
Lymphoma & 10 & 32.3 \\
Germ cell tumor & 5 & 16.1 \\
Bronchogenic carcinoma lympadenopathy & 2 & 6.5 \\
Metastases & 1 & 3.2 \\
\hline
\end{tabular}

Table 2. Middle mediastinal lesion distribution.

\begin{tabular}{|c|c|c|}
\hline Type of lesion & Count & Percentage \\
\hline Bronchogenic cyst & 1 & 4.5 \\
\hline Bronchogenic carcinoma lymphadenopathy & 7 & 31.8 \\
\hline Granulocytic sarcoma & 1 & 4.5 \\
\hline Lymphoma & 7 & 31.8 \\
\hline Metastases & 3 & 13.6 \\
\hline Sarcoidosis & 3 & 13.6 \\
\hline
\end{tabular}

Table 3. Posterior mediastinal lesion distribution.

\begin{tabular}{cccc} 
Type of lesion & Count & Percentage & 33.3 \\
Oesophageal carcinoma & 3 & 1 & 11.1 \\
Oesophageal leiomyoma & 4 & 44.4 \\
Paravertebral abscess & 1 & 1.1 \\
Metastases & & 4 \\
\hline
\end{tabular}


In our study, thymic lesions constituted 14\% .Our study is similiar to study done by Davis et al. in 400 cases, where the thymic masses contributed $19.4 \%$ [5]. In another study conducted by Cohen et al. in 230 cases, $24.3 \%$ were thymic masses [6]. In our study, all the thymic lesions were seen in anterior mediastinum. Among thymic lesions, majority were thymoma (62.5\%) (Figure 2). Remaining thymic lesions were invasive thymoma (12.5\%), thymic carcinoma $(12.5 \%)$ and hemorrhagic thymic cyst $(12.5 \%)$. In a study by Chen et al. on 34 patients with CT diagnosis of thymic mass, thymoma constituted 91\%, thymiccyst $2.9 \%$ [7]. According to Naidich, thymomais most commonly seen between 50 - 60 years [8]. In our study it was seen between 37 to 74 years. In our study, among the 6 thymoma cases including invasive thymoma, one case had myasthenia gravis (16.6\%). Approximately $30 \%$ - $40 \%$ of thymoma patients have myasthenia gravis and $10 \%$ - 15\% of patient with myasthenia gravis have thymomas [9]. We had one case of invasive thymoma which was predicted correctly on CT. Obliteration of fat planes in the mediastinum, pleural thickening and irregular interface with lung were noted which favoured tumor invasion. Among the 5 thymoma cases, all the lesions were solid. Post contrast images showed $40 \%$ heterogenous, $40 \%$ homogenous and 20\% non enhancement. Mass effect was noted in $60 \%$ of thymoma cases. In our study we had one case of thymiccarcinoma which was predicted on CT as thymoma. On CT though there were no signs of mediastinal invasion or lymph node enlargement, histopathologically it was proved as thymic carcinoma. In our study, we had one rare case of hemorrhagic thymiccyst which was diagnosed on CT as germ cell tumor considering the young age and CT features of cyst with Hyperdense on enhancing component. The non enhacing hyperdense structure within the cyst was blood clot. Predictive power of CT diagnosis of thymoma against histopathology diagnosis was tried. CT diagnosis of thymoma had 100\% sensitivity, 97.8\% specificity and 98\% accuracy. Marjolin et al., did a retrospective study to analyse the accuracy of CT of the mediastinum in predicting the histological findings in patients with myasthenia gravis referred for thymectomy [10]. The average sensitivity of CT imaging in their study population was $75 \%$ and the average specificity was $62 \%$. Their study concluded that, CT is helpful in detecting thymoma in patients with myasthenia gravis referred for thymectomy.

In our study of 50 cases of mediastinal lesions, 10 cases (20\%) were lymphoma which is similar to study conducted by Wychulis et al. [11] (i.e. 10.1\%). The lymphoma population in our series was similar to that of the Mayo Clinic. 60\% (6 out of 10) of our patients had Hodgkin disease as opposed to 59\% at the Mayo Clinic [11]. In our study, CT appearance of mediastinal lymphoma were of the three types as follows: 1) multiple enlarged lymph nodes conglomerated to each other and giving a lobulated contour. 2) Large solid mass insinuating and infiltrating the mediastinal vessels with mass effect. 3) Single mass with maintained mediastinal fat plane. Associated findings of multiple axillary lymph nodes, internal mammary lymph nodes, paracardiac lymph nodes favoured CT diagnosis of lymphoma. Totanarungroj et al., in a retrospective study concluded that anterior mediastinal mass associated with lymphadenoapathy was significantly found in lymphoma (75\%) compared to other tumors [12]. Predictive power of CT diagnosis of mediastinal lymphoma (Figure 3) against histopathology diagnosis was tried. CT diagnosis of lymphoma had 100\% sensitivity, 95\% specificity and 96\% accuracy.

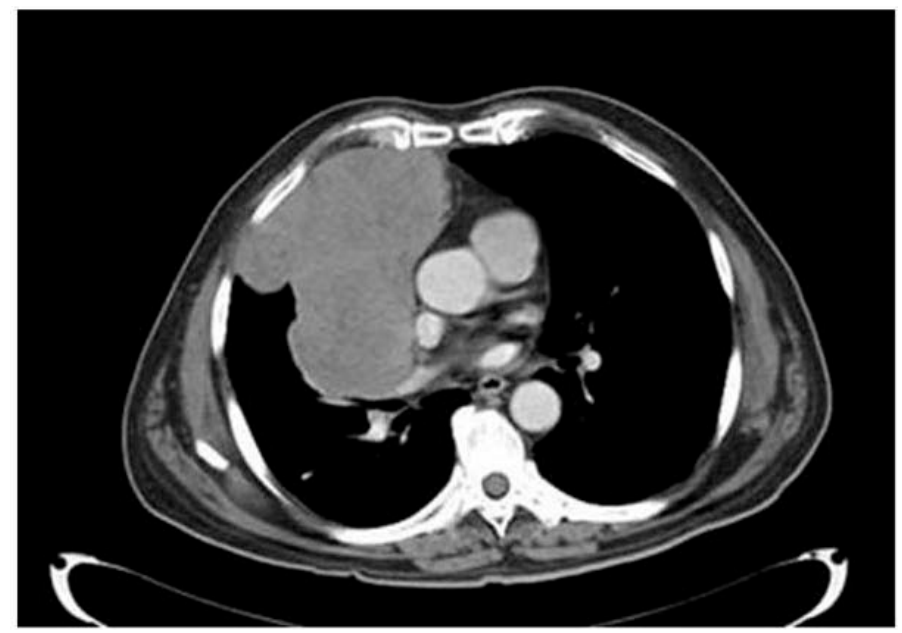

Figure 2. Axial CECT chest (mediastimal window) shows a soft tissue density mass lesion seen in anterior mediastimum (thymoma). 


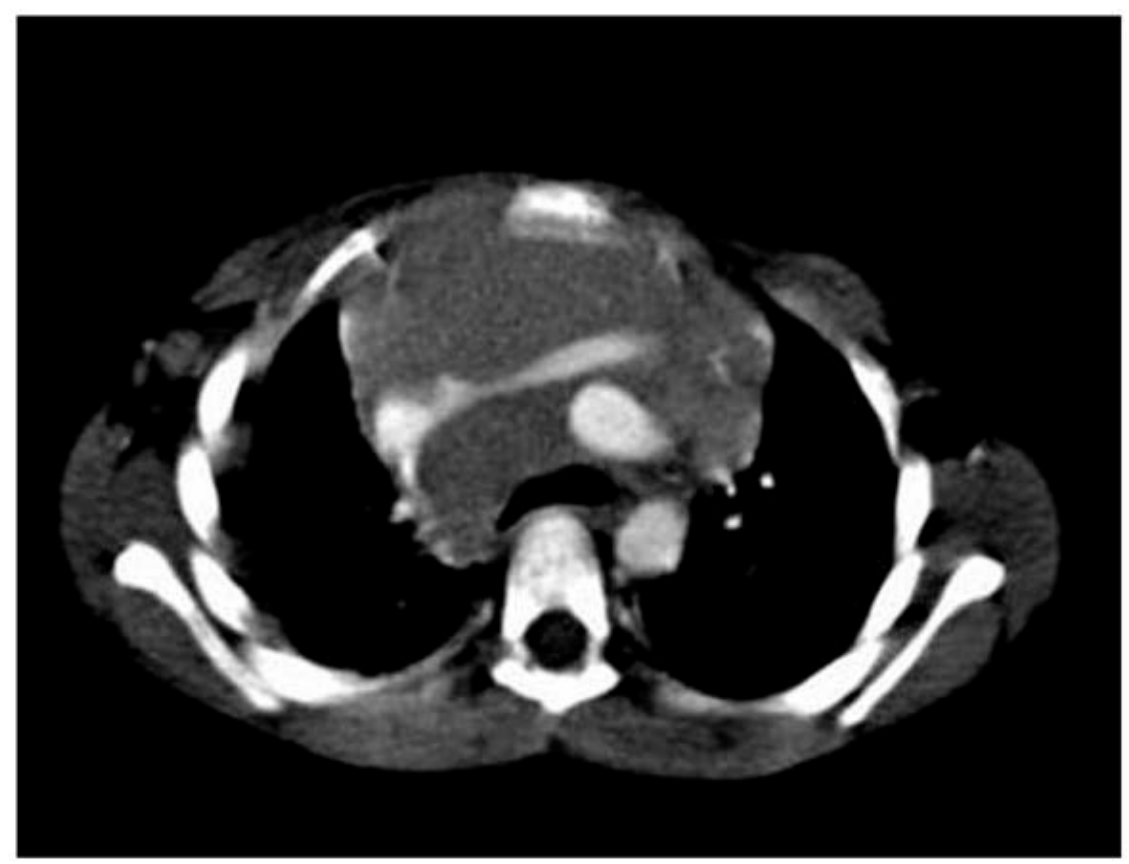

Figure 3. Axial CECT chest (medisatinal window) shows a soft tissue mass encasing the greater vessels.

Totanarungroj et al. in their study concluded that fat density within anterior mediastinal mass was present in 57.1\% germ cell tumor which was significantly higher than other anterior mediastinal tumors [12]. In our study, calcification was seen in $60 \%$ of cases. $60 \%$ of germ cell tumor showed heterogenous enhancement. Mass effect and pleural involvement was seen in $40 \%$ and $20 \%$ respectively. In our study among the 5 cases of mediastinal germ cell tumors, one case was teratoma and remaining were non teratomatous germ cell tumors of the mediastinum. Tian et al. in their study concluded that the characteristic CT findings of primary non-teratomatous germ cell tumors of the mediastinum include bulky, ill-circumscribed, lobulated masses, heterogeneous attenuation with low-attenuation foci and calcification on non-enhanced CT images and heterogeneous enhancement after contrast administration [13]. Predictive power of CT diagnosis of mediastinal germ cell tumor against histopathology diagnosis was tried. CT diagnosis of germ cell tumor had $80 \%$ sensitivity, $97.8 \%$ specificity and $96 \%$ accuracy.

In our study we had 7 cases (14\%) of bronchogenic carcinoma lymph adenopathy presenting as mediastinal lesion. Most of them were multiple. $66.7 \%$ were seen in anterior mediastinum and $100 \%$ in middle mediastinum. Post contrast images revealed homogenous enhancement in 50\%, heterogenous enhancement in $16.7 \%$ and peripheral enhancement in 33.3\% of cases. Majority of mediastinal bronchogenic carcinoma lymphadenopathy lesions were associated with the primary lesion in lung parenchyma, which was identified in CT. Predictive power of CT diagnosis of mediastinal bronchogenic carcinoma lymphadenopathy against histopathology diagnosis was tried. CT diagnosis of bronchogenic carcinoma lymphadenopathy had 85.7\% sensitivity, 100\% specificity and $98 \%$ accuracy. In our study there were 3 cases of metastatic mediastinal lesions other than bronchogenic carcinoma lymphadenopathy. They were predicted on CT correctly (100\% sensitivity, 100\% specificity) as they had a known history of primary lesion. Primary lesions were from renal cell carcinoma, neuroendocrine tumor and neuroblastoma. Murray et al. [2] in their CT series, concluded that metastatic mediastinal lesions contributed to $42 \%$ of mediastinal masses.

In our study we had 3 cases (6\%) of multinodular goiter (Figure 4 ) presenting as mediastinal lesion. In a selected series, mediastinal goiters have constituted 5\% - 11\% of mediastinal masses resected at thoracotomy [14]. In our study, all the 3 cases were predicted correctly on CT. Predictive power of CT diagnosis of multinodular goiter against histopathology diagnosis was tried. CT diagnosis of multinodular goiter had 100\% sensitivity, 100\% specificity and $100 \%$ accuracy. We feel that, in most instances, CT scanning is capable of specifically identifying mediastinal goiter as the cause of a mediastinal mass noted on chest radiography. In each of our cases, the mediastinal thyroid was in direct continuity with tissue originating from the cervical thyroid. Although this was 


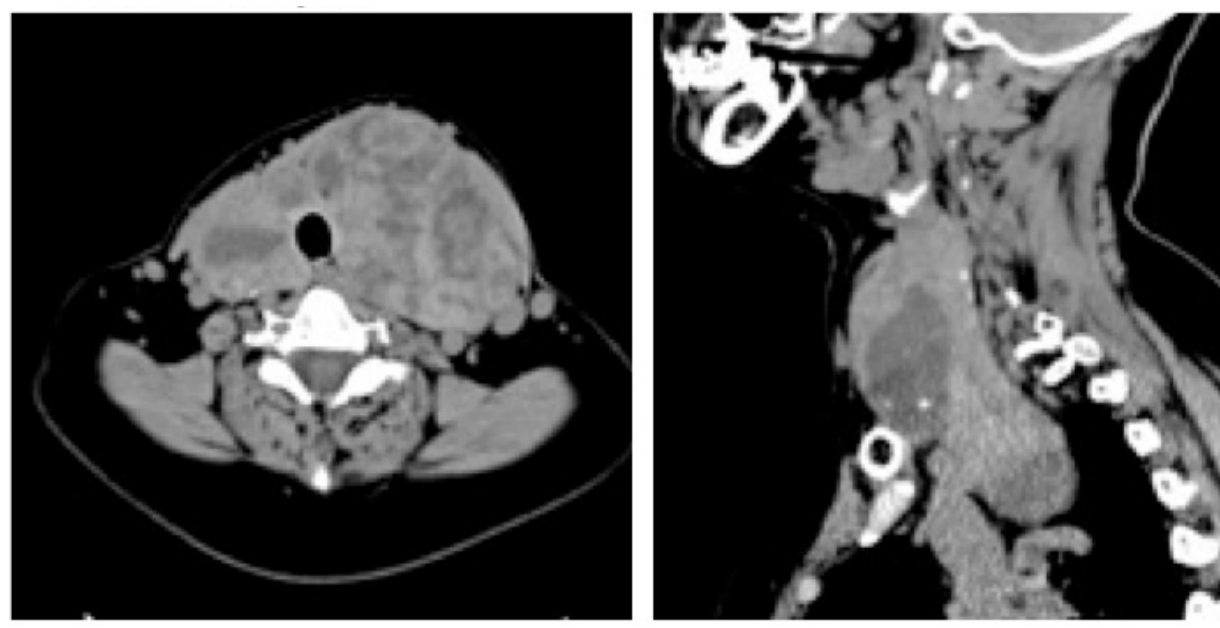

Figure 4. NECT neck, axial sections shows diffuse thyromegaly with heterogeneous mass (multinodular goiter); Sagittal MPR neck shows the retrosternal extension.

evident on axial images, reconstructed images in the coronal plane could be used to more easily verify the continuity in difficult cases. Thus, in evaluation of mediastinal masses, we recommend that scanning be carried up to the neck if more caudad scans indicate the possibility of substernal thyroid as the cause of the mediastinal mass. In our study all the 3 cases presented as anterior mediastinal mass. Considering the nature, $100 \%$ had solid component and $66.7 \%$ had cystic component and $100 \%$ had calcification. All the 3 cases showed heterogenous enhancement (100\%). A factor that helps identify mediastinal tissue as thyroid relates to the high attenuation value of the tissue. CT attenuation greater than muscle attenuation is unusual for mediastinal masses such as lymphoma or thymoma. In our study we had one rare case of thyroid lymphoma presenting as anterior mediastinal mass in a old age female patient. On CT it was seen as anterior mediastinal lesion in continuity with cervical thyroid. It was solid and cystic in nature with calcification. Post contrast images showed heterogenous enhancement. It was associated with cervical lymphadenopathy. We had given a diagnosis of thyroid carcinoma and histopathology diagnosis was Non hodgkin's lymphoma of thyroid. Kim et al. in their study concluded that primary thyroid lymphoma should be included in the differential diagnosis when old female with history of rapidly enlarging thyroid mass and coexistent Hashimoto's thyroiditis had CT findings of homogeneous thyroidal mass isoattenuating to muscles without invasion of surrounding structures [15].

In our study of 50 cases of mediastinal lesions, we had 3 cases (6\%) of oesophageal carcinoma and 1 case of oesophageal leiomyoma presenting as posterior mediastinal mass. All 4 oesophageal lesions were predicted correctly. In our study oesophageal carcinoma, showed homogenous enhancement (66.7\%) and heterogenous enhancement (33.7\%). Among the 3 cases of oesophageal carcinoma, none showed mediastinal invasion. The main purpose of CT in patients with esophageal carcinoma is to stage the disease as accurately as possible in order to determine which patients may be suitable for surgical resection. The reported accuracy of CT in diagnosing mediastinal invasion ranges between 59\% and 82\% [16]. In our study oesophageal leiomyoma presented as posterior mediastinal mass on chest radiograph. On further evaluation with CT, esophageal leiomyoma was predicted. On CT it appeared as well defined eccentric intramural mass in middle third of esophagus with non enhancement on post contrast images. Lesion showed no mediastinal invasion. A factor that helped us to identify the lesion as leiomyoma, was the coarse amorphous calcification on the intramural mass. Leiomyomas may contain amorphous areas of calcification and a calcified esophageal mass should strongly suggest the diagnosis of a leiomyoma [17].

In our study of 50 cases of mediastinal lesions, we had 4 cases of paravertebral abscess presenting as posterior mediastinal mass. All the 4 cases were predicted correctly on CT (100\% sensitivity, $100 \%$ specificity). Among the 4 cases all were cystic (100\%) with spine involvement (100\%) and showed peripheral enhancement (100\%) on post contrast images. Among the 4 cases, $50 \%$ were pyogenic and $50 \%$ were tuberculous. Anterior vertebral end plate erosions were noted in all the 4 cases.

In our study of 50 cases of mediastinal lesions, we had 3 cases of sarcoidosis presenting as lymph node masses in middle mediastinum. All the three cases were predicted correctly on CT (100\% sensitivity, $100 \%$ spe- 
cificity). All the 3 cases had bilateral hilar and paratracheal lymphnodes with homogenous post contrast enhancement. None of the cases had lung parenchymal involvement. We had one case of bronchogenic cyst presenting as non enhancing middle mediastinal cyst in sub carinal location. It was predicted correctly on CT due to its typical CT characteristics and location. In our study we had an unusual case of granulocytic sarcoma presenting as anterior and middle mediastinal mass which was diagnosed histopathologically. We had made a CT diagnosis of lymphoma as it appeared as a heterogenously enhancing solid lesion with cystic areas on CT. Takasugi et al. in their study concluded that mediastinum was common location among intrathoracic granulocytic sarcoma [18].

In our study among the 50 cases of mediastinal lesions, 44 cases (88\%) were predicted correctly by CT when compared with histopathology diagnosis.

\section{Conclusion}

MDCT is a very useful modality for evaluating the distribution and involvement of neighbouring structures by the mediastinal lesions. Overall, MDCT appears to be the best study as it provides information regarding the size, contour, extent, tissue composition, lesion enhancement pattern, airway invasion, mediastinal and vascular invasion. Post-processing techniques further improve the diagnosis and surgical planning by better depicting the anatomy and reducing radiologist fatigue. In our study, we used varying CT features of each mediastinal pathology types to predict a histological diagnosis of mediastinal lesions. Most of the lesions could be predicted with good sensitivity, specificity and diagnostic accuracy.

\section{References}

[1] Benjamin, S.P., McCormack, L.J., Effler, D.B. and Groves, L.K. (1972) Primary Tumors of the Mediastinum. Chest, 62, 297-303. http://dx.doi.org/10.1378/chest.62.3.297

[2] Rebner, M., Gross, B.H., Robertson, J.M., Pennes, D.R., Spizarny, D.L. and Glazer, G.M. (1987) CT Evaluation of Mediastinal Masses. Computerized Radiology, 11, 103-110. http://dx.doi.org/10.1016/0730-4862(87)90033-3

[3] Strollo, D.C., Rosado-de-Christenson, M.L. and Jett, J.R. (1997) Primary Mediastinal Tumors. Part 1. Tumors of the Anterior Mediastinum. Chest, 112, 511-522. http://dx.doi.org/10.1378/chest.112.2.511

[4] Takeda, S., Miyoshi, S., Akashi, A., Ohta, M., Minami, M., Okumura, M., Masaoka, A. and Matsuda, H. (2003) Clinical Spectrum of Primary Mediastinal Tumors: A Comparison of Adult and Pediatric Populations at a Single Japanese Institution. Journal of Surgical Oncology, 83, 24-30. http://dx.doi.org/10.1002/jso.10231

[5] Davis, R.D., et al. (1987) Primary Cysts and Neoplasms of the Mediastinum: Recent Changes in Clinical Presentations, Methods of Diagnosis, Management and Results. Annals of Thoracic Surgery, 44, 229-237. http://dx.doi.org/10.1016/S0003-4975(10)62059-0

[6] Cohen, A.J., Thompson, L.N., Edwards, F.H., et al. (1991) Primary Cysts and Tumors of the Mediastinum. Annals of Thoracic Surgery, 51, 378-386. http://dx.doi.org/10.1016/0003-4975(91)90848-K

[7] Chen, J., Weisbrod, G.L. and Herman, S.J. (1988) Computed Tomography and Pathologic Correlations of Thymic Lesion. Journal of Thoracic Imaging, 3, 61-65. http://dx.doi.org/10.1097/00005382-198801000-00010

[8] Naidich, D.P., Webb, W.R., Muller, N.L., Zerhouni, E.A. and Seigelmann, S.S. (1999) Mediastinum. Chapter 2. In: Naidich, D.P., Muller, N.L., Zerhouni, E.A., Webb, W.R. and Krinsky, G.A., Eds., Computed Tomography and Magnetic Resonance of the Thorax, 3rd Edition, Lippincott Williams and Wilkins, Philadelphia, 38-160.

[9] Lara Jr., P.N. (2000) Malignantthymoma: Current Status and Future Directions. Cancer Treatment Reviews, 26, 127131. http://dx.doi.org/10.1053/ctrv.1999.0159

[10] de Kraker, M., et al. (2005) CT and Myasthenia Gravis: Correlation between Mediastinal Imaging and Histopathological Findings. Interactive CardioVasc Thoracic Surgery, 4, 267-271. http://dx.doi.org/10.1510/icvts.2004.097246

[11] Wychulis, A.R., Payne, W.S., Clagett, O.T., et al. (1971) Surgical Treatment of Mediastinal Tumors: A 40 Year Experience. Journal of Thoracic and Cardiovascular Surgery, 62, 379-392.

[12] Totanarungroj, K., Watcharaporn, C. and Muangman, N. (2010) Helpful CT Findings for Giving Specific Diagnosis of Anterior Mediastinal Tumors. Journal of the Medical Association of Thailand, 93, 489-496.

[13] Tian, L., Liu, L.Z., Cui, C.Y., Zhang, W.D. and Kuang, Y.L. (2012) CT Findings of Primary Non-Teratomatous Germ Cell Tumors of the Mediastinum-A Report of 15 Cases. European Journal of Radiology, 81, 1057-1061. http://dx.doi.org/10.1016/j.ejrad.2011.02.005

[14] Binder, R., Pugatch, R., Faling, L., Kanter, R. and Sawin, C. (1980) Diagnosis of Posterior Mediastinal Goiter by Com- 
puted Tomography. Journal of Computer Assisted Tomography, 4, 550-552. http://dx.doi.org/10.1097/00004728-198008000-00031

[15] Kim, H.C., Han, M.H., Kim, K.H., Jae, H.J., Lee, S.H., Kim, S.S., Kim, K.H. and Chang, K.H. (2003) Primary Thyroid Lymphoma: CT Findings. European Journal of Radiology, 46, 233-239. http://dx.doi.org/10.1016/S0720-048X(02)00134-1

[16] Rankin, S. (1998) Oesophageal Cancer. In: Husband, J.E.S. and Reznek, R.H., Eds., Imaging in Oncology, Isis Medical Media, Oxford, 93-110.

[17] Megibow, A.J., Balthazar, E.J., Hulnick, D.H., et al. (1985) CT Evaluation of Gastrointestinal Leiomyomas and Leiomyosarcomas. AJR, 144, 727-731. http://dx.doi.org/10.2214/ajr.144.4.727

[18] Takasugi, J.E., Godwin, J.D., Marglin, S.I. and Petersdorf, S.H. (1996) Intrathoracic Granulocytic Sarcomas. Journal of Thoracic Imaging, 11, 223-230. http://dx.doi.org/10.1097/00005382-199601130-00006 